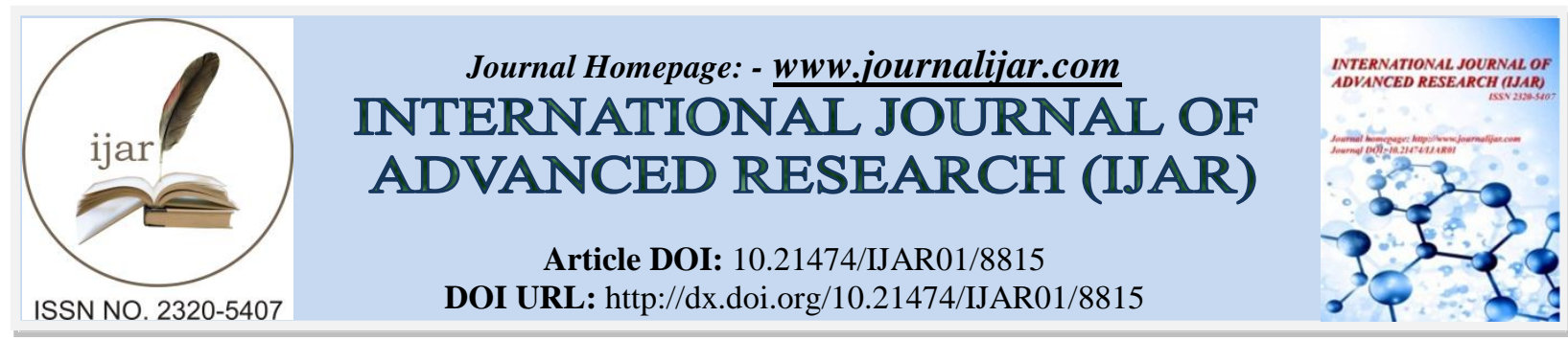

RESEARCH ARTICLE

\title{
WERNICKE ENCEPHALOPATHY COMPLICATING GASTRECTOMY IN A PATIENT WITH A HISTORY OF GASTRIC ADENOCARCINOMA.
}

Sanae Chaoui, Fatima Zahra Laamrani and Leila Jroundi.

Service de Radiologie des urgences, centre hospitalier universitaire Ibn Sina - Rabat- Maroc.

\section{Manuscript Info}

Manuscript History

Received: 05 February 2019

Final Accepted: 07 March 2019

Published: April 2019

Key words:-

Wernicke encephalopathy, thiamine, thalami

\begin{abstract}
Wernicke encephalopathy (WE) is a severe neurologic disorder caused by thiamine deficiency, characterized by ataxia, ophthalmoplegia, nystagmus, and mental confusion. It is typically seen in alcoholics. On imaging, it is commonly seen on MRI as areas of symmetrical increased T2/FLAIR signal involving the mammillary bodies, dorsomedial thalami, tectal plate, periaqueductal area, and around the third ventricle. The early treatment avoids the installation of a korsakoff psychosis. We report a case of a patient of 57 years old without a history of alcohol abuse, who developed Wernicke syndrome 6 months after undergoing gastrectomy for gastric adenocarcinoma.
\end{abstract}

Copy Right, IJAR, 2019,. All rights reserved.

\section{Introduction:-}

L'encéphalopathie de Gayet-Wernicke est une encéphalopathie carentielle grave liée à un déficit en thiamine (vitamine B1), fréquemment rencontrée chez les grands consommateurs d'alcool, et de manière plus générale observée dans tout contexte de dénutrition sévère. Cliniquement, il se révèle par une triade incluant des troubles oculomoteurs, une ataxie cérébelleuse et un syndrome confusionnel. C'est une urgence médicale, dont les séquelles risquent d'être importantes en l'absence de traitement, ce dernier ne doit pas être retardé par les dosages vitaminiques. Nous rapportons un cas d'encéphalopathie de Gayet-Wernicke compliquant une gastrectomie réalisée chez un patient de 57 ans aux antécédents d'adénocarcinome gastrique.

\section{Observation:-}

Il s'agit d'un patient âgé de 57 ans, ayant bénéficié d'une gastrectomie totale pour adénocarcinome gastrique avec chimiothérapie adjuvante. Il n'y a pas de notion de prise toxique ou d'éthylisme. Le patient s'est présenté dans un tableau fait de trouble de conscience avec troubles oculomoteurs remontant à 10jours. L'examen clinique a retrouvé un patient confus, avec paralysie des nerfs oculomoteurs et nystagmus horizontal. Un scanner cérébral a été réalisé initialement; il est revenu sans particularité. L'IRM cérébrale demandée par la suite a mis en évidence un hypersignal T2, FLAIR et diffusion des noyaux médians des thalami (fig. 1, 2 et 4) et des corps mamillaires (fig. 3 et 5) de façon bilatérale et symétrique, associé à un discret hypersignal FLAIR péri-acqueducal (fig. 3). Le diagnostic d'encéphalopathie de Gayet-Wernicke a été posé, confirmé par le dosage de la vitamine B1 qui était à $21 \mathrm{nmol} / 1$, et la supplémentation vitaminique a été démarrée chez notre patient.

\section{Discussion:-}

L'encéphalopathie de Gayet-Wernicke est une encéphalopathie carentielle grave liée à un déficit aigu et massif en vitamine B1 (la thiamine). Elle a été décrite pour la première fois en 1881 par Carl Wernicke chez un homme alcoolique et une femme présentant des vomissements incoercibles (1). 
La thiamine est une vitamine hydrosoluble d'origine uniquement alimentaire. L'organisme en possède un stock limité d'environ 25-30 mg (7). Les besoins journaliers de thiamine sont d'environ 1,1 à 1,2 mg/jour (7), augmentant chez la femme enceinte et qui allaite. En cas de manque d'apport, le déficit apparaît déjà après 2 à 3 semaines. L'absorption de la thiamine se fait au niveau du duodénum par un processus actif (7). Elle traverse la barrière hémato-méningée de façon active et surtout passive en fonction du gradient de concentration. Sa forme biologique active est le pyrophosphate de thiamine qui est un coenzyme essentiel de plusieurs réactions biochimiques au niveau du cerveau. Ainsi, le déficit en thiamine entraine au niveau cerebral des lésions de sévérité variable, allant des suffusions hémorragiques à la destruction de neurons $(1,5)$. Sur le plan anatomopathologique, il associe œdème, nécrose, démyélinisation, perte neuronale et dilatation artério-capillaire (5).

L'encéphalopathie de Gayet-Wernicke s'installe de façon rapidement progressive, plus fréquemment sur un terrain d'alcoolisme chronique, mais de manière plus générale dans tout contexte de dénutrition sévère. Ainsi, les facteurs favorisants sont les vomissements gravidiques, l'alimentation parentérale prolongée, les tumeurs gastro-intestinales, la chimiothérapie, la chirurgie digestive, l'insuffisance rénale chronique et l'anorexie mentale (1).Chez notre patient, plusieurs facteurs favorisaient la survenue de l'encéphalopathie de Gayet-Wernicke: le cancer gastrique, la gastrectomie et la chimiothérapie, sans supplémentation vitaminique.

Cliniquement, elle se révèle par une triade qui n'est complète que dans $30 \%$ des cas, incluant des troubles psychiques avec syndrome confusionnel et troubles mnésiques, des troubles oculomoteurs à type de paralysies oculomotrices et nystagmus, et une ataxie cérébelleuse (8).

Devant une suspicion d'encéphalopathie de Gayet-Wernicke, l'IRM constitue l'examen de référence.
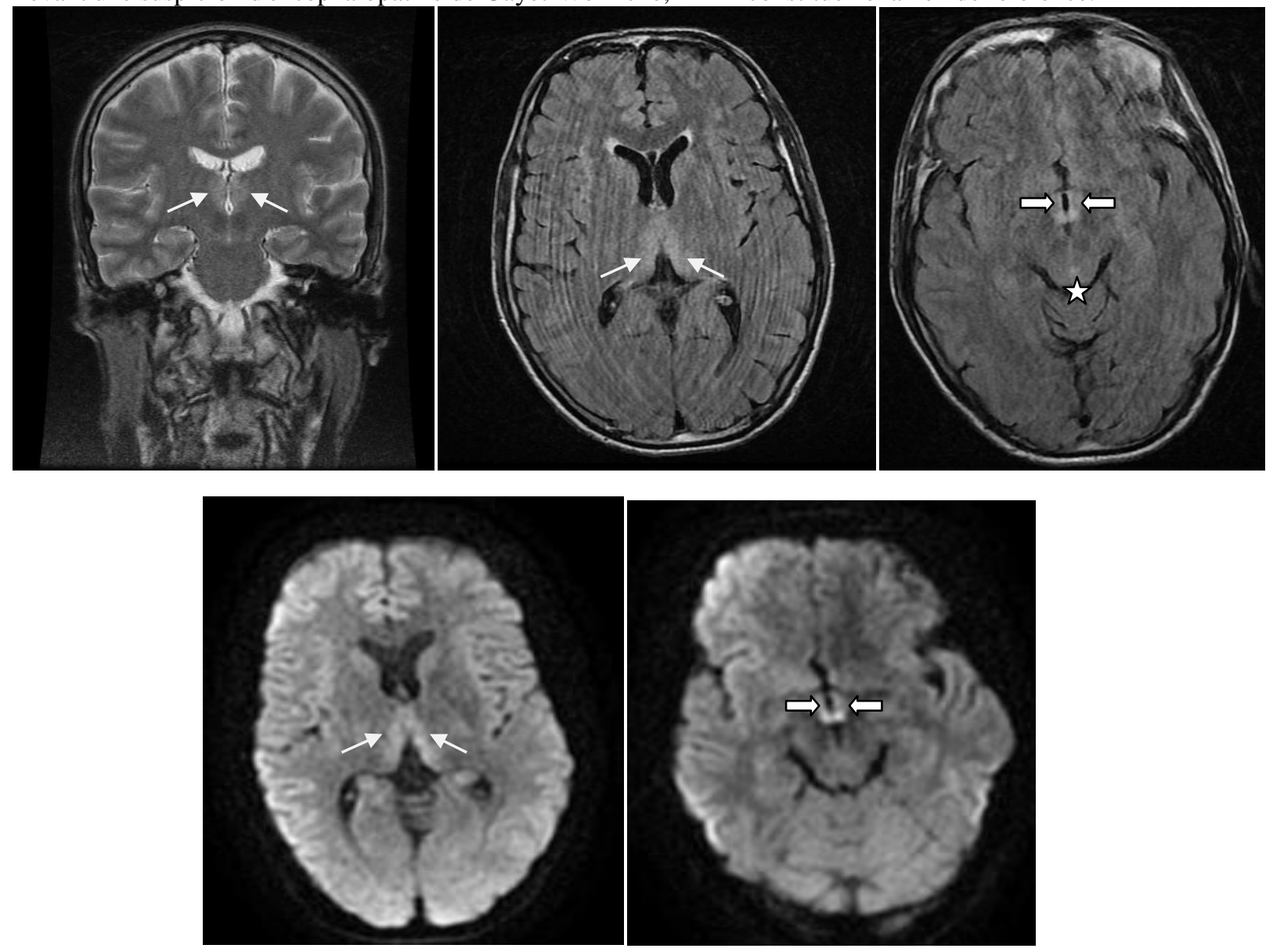

Fig 1, 2, 3, 4 et 5: IRM cérébrale en séquences pondérées T2 (coronale), FLAIR (axiales), et diffusion (axiales): Hypersignal T2, FLAIR et diffusion bilatéral et symétrique des noyaux médians des thalami (flèches) et des corps mamillaires (flèches pleines), avec discret hypersignal FLAIR péri-acqueducal (astérix). 
Elle est caractérisée par des lésions de topographie thalamique, avec une atteinte bilatérale et symétrique des noyaux postéro-médians. Elle peut s'associer à d'autres lésions comme celle des corps mamillaires, la région périacqueducale, le plancher du V4, autour du V3, et le vermis (1).

Bien que très évocatrices, ces anomalies de signal ne sont pas pathognomoniques (spécificité de $93 \%$ ), Il faut garder à l'esprit les principaux diagnostics différentiels (accident vasculaire cérébral, thrombose veineuse profonde, maladie de Creutzfeldt-Jakob, encéphalite à Cytomégalovirus, lymphome) (2).

Le traitement doit être précoce, dès la suspicion diagnostique et ne doit pas être retardé par les dosages vitaminiques. Il n'y a pas de consensus sur la quantité de thiamine à délivrer et la durée de traitement. Toutefois, l'European Federation of Neurological Societies recommande 1'administration de thiamine à la dose de 200 mg trois fois par jour, de préférence par voie intraveineuse avant toute administration de glucose et reprise d'une alimentation normale (9). Le traitement devra être poursuivi jusqu'à ce qu'il y ait une amélioration des signes (9).

L'évolution est en règle favorable sous vitaminothérapie précoce, avec régression ou disparition des anomalies de signal, et dilatation secondaire du V3 et de l'aqueduc de Sylvius (2). L'évolution favorable des images n'est pas corrélée à l'évolution clinique (2). Si l'ophtalmoplégie régresse dans les heures suivant l'instauration du traitement, l'ataxie peut être plus longue à récupérer (séquelles dans $25 \%$ des cas), et des séquelles psychomnésiques plus ou moins graves peuvent être notées (2). En revanche, en cas de retard diagnostique et/ou thérapeutique l'évolution peut être défavorable marquée par la survenue du syndrome de Korsakoff révélé cliniquement par des troubles de la mémoire chroniques et une anosognosie, avec atrophie importante des corps mamillaires à l'imagerie $(1,2)$.

La prévention passe par une administration parentérale de vitamine B1 $(250 \mathrm{mg}$ en intramusculaire pendant 3 à 5 jours) à tous les patients à risqué (9).

\section{Conclusion:-}

L'encéphalopathie de Gayet-Wernicke est une pathologie qui reste sous-diagnostiquée, en particulier lors d'étiologies non alcooliques. Devant une situation à risque, notamment lors de tout contexte de dénutrition, la triade ophtalmoplégie, confusion mentale et ataxie cérébelleuse est évocatrice et doit faire penser au diagnostic. L'IRM constitue l'examen de référence pour l'exploration de cette entité, et le dosage vitaminique ne doit pas retarder l'instauration du traitement, afin d'éviter l'évolution vers des complications plus graves.

\section{Bibliographie:-}

1. Diallo1 I, Boury S Gning, Ndiaye A, Ndiaye B, Dieng M, Diémé Y, Mbaye P.S. Encéphalopathie de GayetWernicke compliquant des vomissements sur terrain de néoplasie colique. Pan African Medical Journal. 2015; 21:179 doi:10.11604/pamj.2015.21.179.4486

2. Koob M, Dietemann J.L. Encéphalopathie de Gayet-Wernicke. Presse Med. 2006; 35: 1095-6.

3. Shimomura T, Mori E, Hirono N, et alToru Imamura, Yamashita H, MA. Development of Wernicke-Korsakoff Syndrome After Long Intervals Following Gastrectomy. ArchNeurol. 1998;55(9):1242-1245. doi:10-1001

4. MR Imaging Findings in 56 Patients with Wernicke Encephalopathy: Non alcoholics May Differ from Alcoholics. Jan2009. AJNR Am J Neuroradiol30:171-76.

5. Zuccoli G, Pipitone N. Neuroimaging Findings in Acute Wernicke's Encephalopathy: Review of the Literature. AJR 2009; 192:501-508.

6. S. Lescuyer - M. Rondeau-Lutz - C. Martinez - P. Bilbault · J.C. Weber. Wernicke's encephalopathy in nonalcoholic patients. Ann. Fr. Med. Urgence (2015) 5:37-42 DOI 10.1007/s13341-014-0499-y

7. Farquet V, Alvarez V, Biselx S et Coutaz M. Rev Med Suisse 2017; 13 : 382-4. Du déficit en thiamine à l'encéphalopathie de Gayet-Wernicke, pathologie méconnue.

8. Young-Chul Jung,Chanraud S, and Edith V. Sullivan. Neuroimaging of Wernicke's Encephalopathy and Korsakoff's Syndrome. Neuropsychol Rev . 2012 June ; 22(2): 170-180. doi:10.1007/s11065-012-9203-4.

9. Galvin R, Brathen G, Ivashynka A, Hillbom M et al. EFNS guidelines for diagnosis, therapy and prevention of Wernicke encephalopathy. Eur J Neurol. 2010;17(12):1408-18. 